\title{
Diagnostic Allele-Specific PCR for the Identification of Candida auris Clades
}

\author{
Hans Carolus ${ }^{1,2}$, Stef Jacobs ${ }^{1}$, Celia Lobo Romero ${ }^{1,2}$, Quinten Deparis ${ }^{2,3}$, Christina A. Cuomo ${ }^{4}$, \\ Jacques F. Meis ${ }^{5,6}\left(\mathbb{1}\right.$ and Patrick Van Dijck ${ }^{1,2, *}$
}

1 Laboratory of Molecular Cell Biology, Department of Biology, Institute of Botany and Microbiology, KU Leuven, 3001 Leuven, Belgium; hans.carolus@kuleuven.be (H.C.); stef.jacobs@student.kuleuven.be (S.J.); celia.loboromero@kuleuven.be (C.L.R.)

2 VIB-KU Leuven Center for Microbiology, 3001 Leuven, Belgium; quinten.deparis@kuleuven.be

3 Laboratory for Genetics and Genomics, Centre for Microbial and Plant Genetics, KU Leuven, 3001 Leuven, Belgium

4 Broad Institute of MIT and Harvard, Cambridge, MA 02142, USA; cuomo@broadinstitute.org

5 Department of Medical Microbiology and Infectious Diseases, Canisius-Wilhelmina Hospital, 6532 Nijmegen, The Netherlands; j.meis@cwz.nl

6 Centre of Expertise in Mycology Radboudumc/CWZ, 6532 Nijmegen, The Netherlands

* Correspondence: patrick.vandijck@kuleuven.be

check for updates

Citation: Carolus, H.; Jacobs, S.; Lobo Romero, C.; Deparis, Q.; Cuomo, C.A.; Meis, J.F.; Van Dijck, P. Diagnostic Allele-Specific PCR for the Identification of Candida auris Clades. J. Fungi 2021, 7, 754. https://doi.org/ 10.3390/jof7090754

Academic Editor: Spinello Antinori

Received: 19 August 2021

Accepted: 10 September 2021

Published: 13 September 2021

Publisher's Note: MDPI stays neutral with regard to jurisdictional claims in published maps and institutional affiliations.

Copyright: (c) 2021 by the authors. Licensee MDPI, Basel, Switzerland. This article is an open access article distributed under the terms and conditions of the Creative Commons Attribution (CC BY) license (https:// creativecommons.org/licenses/by/ $4.0 /)$.
Abstract: Candida auris is an opportunistic pathogenic yeast that emerged worldwide during the past decade. This fungal pathogen poses a significant public health threat due to common multidrug resistance (MDR), alarming hospital outbreaks, and frequent misidentification. Genomic analyses have identified five distinct clades that are linked to five geographic areas of origin and characterized by differences in several phenotypic traits such as virulence and drug resistance. Typing of $C$. auris strains and the identification of clades can be a powerful tool in molecular epidemiology and might be of clinical importance by estimating outbreak and MDR potential. As C. auris has caused global outbreaks, including in low-income countries, typing C. auris strains quickly and inexpensively is highly valuable. We report five allele-specific polymerase chain reaction (AS-PCR) assays for the identification of $C$. auris and each of the five described clades of $C$. auris based on conserved mutations in the internal transcribed spacer (ITS) rDNA region and a clade-specific gene cluster. This PCR method provides a fast, cheap, sequencing-free diagnostic tool for the identification of C. auris, C. auris clades, and potentially, the discovery of new clades.

Keywords: Candida auris; diagnostics; clades; PCR

\section{Introduction}

Candida auris was first described in 2009 [1] and has emerged on three different continents in less than a decade [2]. C. auris is closely related to Candida haemulonii, Candida duobushaemulonii, and Candida pseudohaemulonii, forming a group of emergent, multidrug resistant (MDR) Candida species that are distantly related to C. albicans and C. glabrata [3]. Misidentification of $C$. auris is an alarming and frequently reported problem [4-6]. The commonly used phenotypic/biochemical identification platforms often fail to identify C. auris correctly. Traditional kits such as Vitek 2 YST or API 20C often lead to the misidentification of C. auris as C. haemulonii, C. duobushaemulonii, C. sake, C. famata, C. guilliermondii, C. Iusitaniae, C. parapsilosis, C. intermedia, C. catenulate, Saccharomyces kluyveri, Saccharomyces cerevisiae, Rhodotorula glutinis, or Rhodotorula rubra [4,7].

Currently, the most reliable, efficient, and therefore, recommended methods for $C$. auris identification are matrix-assisted laser desorption ionization time-of-flight mass spectrometry (MALDI-TOF MS) and rDNA sequencing $[4,8]$. The disadvantage of these methods is the cost, high-tech equipment, and skilled labor they require. Recently, several 
PCR and qPCR assays were designed for rapid, cheap, and accurate identification of $C$. auris $[4,9-11]$.

Whole-genome sequencing of $C$. auris isolates has shown that strains from different continents group into five distinct clades associated with a primary geographical distribution: the South Asian clade or 'clade I', the East Asian clade or 'clade II', the South African clade or 'clade III', the South American clade or 'clade IV', and the Iranian clade or 'clade $\mathrm{V}^{\prime}[12,13]$. All $\mathrm{C}$. auris strains, isolated from patients in at least 47 countries, cluster in one of those five clades [14,15]. While the within-clade diversity is limited, thousands of single-nucleotide polymorphisms (SNPs) differentiate the five different clades, sufficient to extrapolate that hundreds of years of divergent evolution separates these genotypes [2]. Different clades have been associated with significantly divergent karyotypes, and mating type loci are clade-specific [3]. Moreover, different clades show clade-specific signatures of selection regarding cell surface manno-proteins [16] and drug-related genes [17]. Overall, this vast genomic diversity between clades translates into clade-specific phenotypes [2,3]. Clade II harbors the greatest percentage of drug-susceptible isolates [2], while clade I has the greatest reported percentage of MDR and pan-resistant isolates and is commonly resistant to fluconazole $[2,13,18]$ and amphotericin B $[2,13]$. Clade III isolates are most often resistant to fluconazole, but resistance to amphotericin B is rare [2,19]. Clade IV isolates have the highest percentage of echinocandin resistance, which only sporadically occurs in clades I and III [2]. Moreover, specific drug resistance-associated mutations have been associated with specific clades [2]. Clade II and V are not associated with hospital outbreaks and commonly cause ear infections, while fungemia is rare $[12,17,20-22]$. Other studies suggest that clade II isolates are more susceptible to disinfection methods [23-25]. Isolates from clade I, III, and IV are, on the other hand, responsible for major hospital outbreaks of frequently multidrug resistant and lethal candidemia [17]. Isolates of clade I, III, and IV are more effective at colonization and dissemination from the gastrointestinal tract, compared to clade II strains in mice [26], while clade I and IV yield higher mortality rates compared to clade II and III in a murine systemic infection model [27]. Another important characteristic with regard to colonization, virulence, persistence in the environment, and thus, nosocomial transmission is biofilm formation [28,29], which also varies significantly between the clades [26,30].

To identify to which clade a strain of $C$. auris belongs, whole-genome sequencing (WGS) is the gold-standard approach $[2,3,12,13,17]$. Several other methods for typing C. auris strains have been investigated, including microsatellite typing, ITS sequencing, AFLP (amplified fragment length polymorphism) fingerprinting, MALDI-TOF MS, and Fourier-transform infrared spectroscopy [31]. Nevertheless, only microsatellite typing and STR (short tandem repeat) typing has been proven to be a reliable alternative to WGS for C. auris clade identification [31,32]. Although ITS sequencing was reliable to differentiate three out of four clades, it showed less resolution compared to microsatellite typing, as C. auris clade I and clade III show no variation within the ITS region [31]. Typing of $C$. auris clades has a role in molecular epidemiology, allowing tracing the origins of nosocomial transmission $[2,19,32,33]$, while this might also confer a clinical value, as it can help to estimate virulence, transmission, and resistance tendencies [2,33].

AS-PCR can be used to verify specific single-nucleotide polymorphisms (SNPs) and deletions or insertions (indels). By designing an allele-specific variation at the $3^{\prime}$ end of a PCR primer and incorporating mismatches, amplification is only successful for that allele at a certain (elevated) primer annealing temperature (Ta) range [34]. In doing so, the presence of a specific allele can be screened for reliably, rapidly, cost-efficiently, and without sequencing by AS-PCR. AS-PCR can be used to identify mutations in research [35,36], or as a diagnostic tool [37-40].

In this study, we investigated specific SNPs, indels, and molecular markers to reliably identify C. auris and the five clades currently described. We optimized a straightforward, cheap, fast, and easy-to-interpret PCR assay that relies on allele-specific amplification to identify C. auris and C. auris clades. We developed AS-PCR primers that yield successful 
PCR amplification of a C. auris-specific ITS2 amplicon but are insensitive to the species that C. auris is commonly misidentified as. For clade identification, we designed a duplex PCR that targets a region of ITS1 that is divergent for four of the five described clades (clades I and III have identical ITS sequences), while we used a clade III/V-specific gene cluster to discriminate clades I and III.

This assay can be used as a tool for epidemiological research and is, due to its lowcost and low-tech necessities, ideal for lower budget research settings including developing regions in which C. auris outbreaks have frequently occurred [14,41]. Moreover, clade-associated phenotypic traits such as virulence and drug resistance can make clade diagnostics an important tool for the clinic, by enabling a quick assessment of the risk for resistance-induced treatment failure or proneness to hospital outbreaks. Lastly, our clade diagnostic tool, along with a more in-depth analysis of the ITS region, can serve to identify potential new clades of $C$. auris.

\section{Materials and Methods}

\subsection{Sequence Analysis for Species- and Clade-Specific Allele Selection}

For the identification of $C$. auris, the ITS region was investigated for species-specific SNPs and/or indels. An alignment of 285-709 bp covering the ITS regions of 32 species was created in CLC Main Workbench v8.1 (Qiagen ${ }^{\circledR}$, Hilden, Germany), by using TYPE/reference strain sequences from NCBI GenBank (ncbi.nlm.nih.gov/genbank/ (accessed on 8 February 2021)). Information about all sequences included in this alignment is listed in Table A1 (Appendix A). The selection of species was based on literature concerning the (mis)identification of C. auris $[4,7,42-44]$ and includes most pathogenic Candida species [45]. The ITS alignment was manually searched for $C$. auris-specific regions to design a C. auris specific AS-PCR primer.

For the identification of $C$. auris clades, a 212-214 bp ITS alignment was created that contained 121 sequences: 96 typed C. auris strains from clade I, II, III, and IV as reported by Vatanshenassan et al. [31] (NCBI GenBank accessions MN242989-MN243084), two Iranian clade V strains [21,22] (NCBI GenBank accessions: MW019910.1 and MZ389242), and 23 C. auris strains from an in-house collection of isolates from all five clades. The latter consists of 15 clade I strains, three clade III strains, three clade IV strains, one clade II strain, and one clade V strain and includes at least one typed strain per clade for which whole-genome sequencing data are available (e.g., clade I: B8441, clade II: B11220, clade III: B11221, clade IV: B11244 [2,3,13,17], and clade V [12]: B18474/IFRC2087; see GenBank accessions PRJNA328792 and PRJNA541007, respectively). Strain information of the in-house collection is summarized in Table A2 (Appendix A). This alignment was manually searched for clade-specific regions to design AS-PCR primers. To illustrate clade divergence based on ITS, an UPGMA (unweighted pair group method with arithmetic mean) phylogenetic tree with Jukes-Cantor correction and 1000 bootstraps was created in CLC Main Workbench v8.1 (Qiagen ${ }^{\circledR}$, Hilden, Germany). To differentiate between clade I and III isolates, which have identical ITS sequences, an L-rhamnose-1-dehydrogenase (RHA1) gene was targeted. RHA1 is part of a rhamnose assimilation gene cluster present in clade III but not in clades I, II, and IV [46]. As data on the presence of this gene cluster were lacking for clade $\mathrm{V}$, this sequence was extracted from the clade III C. auris isolate B11221 genome (accession PRJNA328792) and used as a query for an NCBI BLAST ${ }^{\circledR}$ search against the SRA Illumina data of one Iranian clade V isolate B18474/IFRC2087 (SRX5786024) [12]. Reads were assembled in CLC Main Workbench v21.0.3 (Qiagen ${ }^{\circledR}$, Hilden, Germany).

\subsection{Strains and Media}

To test the AS-PCR assay for C. auris identification, a panel of 15 species was used. This panel was composed of species for which the ITS region shows similarities to the C. auris ITS region and/or species that $C$. auris has been misidentified as [4]. This panel contained C. auris (B8441), C. haemulonii (CBS 5149), C. duobushaemulonii (CBS 7798), C. pseudohaemulonii (CBS 10004), C. albicans (SC 5314), C. tropicalis (CBS 94), C. parapsilosis (CBS 604), C. glabrata (CBS 138), C. lusitaniae (CBS 4413), Candida orthopsilosis (CBS 10906), 
Candida metapsilosis (CBS 10907), C. sake (CBS 159), Candida dubliniensis (CD36), Candida krusei (CBS 573), and S. cerevisiae (BY4741).

To test the AS-PCRs for clade identification, a panel of 23 C. auris strains was used. Strain origin, strain reference $\mathrm{nr}$ (if assigned), and strain clade as identified based on ITS sequencing, microsatellite typing, and/or whole-genome sequencing of each $C$. auris strain used in this project are listed in Table A2 (Appendix A).

All strains were grown on yeast peptone dextrose (YPD, $2 \%$ glucose) agar at $30{ }^{\circ} \mathrm{C}$ or $37^{\circ} \mathrm{C}$ and stored in YPD liquid medium containing $25 \%$ glycerol at $-80^{\circ} \mathrm{C}$.

\subsection{DNA Extraction}

Cells were dissolved in $400 \mu \mathrm{L}$ of TE buffer (10 mM Tris (pH 1), 1\% SDS, 1 mM EDTA, $100 \mathrm{mM} \mathrm{NaCl}, 2 \%$ Triton X-100) and $400 \mu \mathrm{L}$ of PCI solution (phenol pH 6.7, chloroform, and isoamyl alcohol in a 25:24:1 ratio) and lysed by micro-bead shearing in a FastPrep ${ }^{\circledR}$ homogenizer ( $30 \mathrm{~s} 6 \mathrm{~m} / \mathrm{s}$ (MP biomedical ${ }^{\mathrm{TM}}$, Brussels, Belgium). The homogenate was centrifuged (10 min, 14,000 rpm), and $300 \mu \mathrm{L}$ of the supernatant was mixed with $30 \mu \mathrm{L}$ of sodium acetate ( $3 \mathrm{M}, \mathrm{pH} 5.2)$ and $900 \mu \mathrm{L}$ of $100 \%$ ethanol. The extract was vortexed, cooled $\left(-20^{\circ} \mathrm{C}\right)$ for $30 \mathrm{~min}$, and centrifuged $(10 \mathrm{~min}, 14,000 \mathrm{rpm})$ at $4{ }^{\circ} \mathrm{C}$ for DNA precipitation. The DNA pellet was washed twice with $500 \mu \mathrm{L}$ of $70 \%$ ethanol, dried at $37^{\circ} \mathrm{C}$, and dissolved in nuclease-free water. The concentration of DNA was measured using a NanoDrop ${ }^{\mathrm{TM}}$ Microvolume spectrophotometer (Thermo Scientific ${ }^{\mathrm{TM}}$, Waltham, MA, USA).

\section{4. $P C R$ and Sequencing}

The ITS region of all C. auris and non-C. auris strains used in this study (see Section 2.2) was sequenced to confirm targeted variable regions. The targeted sequences were amplified by PCR, using Q $5{ }^{\circledR}$ High-Fidelity DNA polymerase (New England Biolabs Inc., Ipswich, MA, USA). The total reaction volume of $50 \mu \mathrm{L}$ contained $500 \mathrm{ng}$ of purified DNA, $5 \mu \mathrm{L}$ of dNTPs $(2.5 \mathrm{mM}), 10 \mu \mathrm{L}$ of $5 \times \mathrm{Q} 5$ buffer, $0.5 \mu \mathrm{L}$ of Q5 polymerase, and $0.4 \mu \mathrm{L}$ of both universal fungal barcoding primers ITS1 and ITS4 $(100 \mu \mathrm{M})$ (Table 1) [47]. The PCR program consisted of initial denaturation at $98^{\circ} \mathrm{C}$ for $30 \mathrm{~s}, 30$ cycles of $98^{\circ} \mathrm{C}$ for $10 \mathrm{~s}, 59^{\circ} \mathrm{C}$ for $25 \mathrm{~s}$, and $72{ }^{\circ} \mathrm{C}$ for $30 \mathrm{~s}$, and a final elongation step at $72{ }^{\circ} \mathrm{C}$ for $2 \mathrm{~min}$ in a Labcycler Basic thermocycler (Bioké, Leiden, The Netherlands). Correct amplification was verified by gel electrophoresis of $5 \mu \mathrm{L}$ of the PCR product on a $1 \%$ agarose gel. Sanger sequencing (TubeSeq service) was performed by Eurofins (Nazareth, Belgium).

Table 1. Primers used in this study. The specific annealing temperature (Ta) is given in ${ }^{\circ} \mathrm{C}$.

\begin{tabular}{ccccc}
\hline Primer Name & Sequence & Use (Ta) & Target & Reference \\
\hline ITS1 & TCCGTAGGTGAACCTGCGG & Sequencing (59) & Fungi & AS-PCR $(68$ or 78) 1 \\
ITS4 & TCCTCCGCTTATTGATATGC & Sequencing (60) & Fungi & [47] \\
ITS_Cau_R & TTTGTGAATGCAACGCCATCG & AS-PCR (78) & C. auris & This study \\
ITS_CauCII_R & GAGATCCGTTGTTGAAAGTTTTCTTTATA & AS-PCR (68) & Clade II & This study \\
RHA1_CauCIII/V_F & TTGCGGTTGAAATGGGTGCT & AS-PCR (68) & Clade III/V & This study \\
RHA1_CauCIII/V_R & TGGCATGTTTCCGGCTTAGA & AS-PCR (68) & Clade III/V & This study \\
ITS_CauCIV/V_R & AGATCCGTTGTTGAAAGTTTTATGTTCTG & AS-PCR (68) & Clade IV/V & This study \\
ITS_CauCV_R & AAGAGATCCGTTGTTGAAAGTTTCCTA & AS-PCR (68) & Clade V & This study \\
\hline
\end{tabular}

${ }^{1} \mathrm{~A}$ Ta of $68^{\circ} \mathrm{C}$ was used for multiplex AS-PCR to identify clades, while a Ta of $78^{\circ} \mathrm{C}$ was used for C. auris species identification.

\subsection{Allele-Specific Primer Design}

Primers for AS-PCR were designed following the method of Liu et al. [34]. All allelespecific primers were designed in silico using CLC Main Workbench v8.1 (Qiagen ${ }^{\circledR}$ ) and are listed in Table 1. To further increase the primer specificity, specific mismatches at the $3^{\prime}$ end of the allele-specific primer were implemented in several primers (see Figure 1). By including additional mismatches, the specificity of the primers for the right allele can be increased at higher annealing temperatures [34]. 
A

C. auris (MN243084.1) 5 ' T C G C G G T G G C G T T G C A T T C A C A A A A T T 2991,2 ITS_Cau_R G C $\mathrm{A}$ C C G C A A C G T A A G T G T T T

C. haemulonii (JX459664.1) 5 ' T T - - G G T G G A T T T G T T T C - - - T A A A T $281^{1}$

C. pseudohaemulonii (EU881972.1) $5^{\prime}$ C A C - G G T G G T T T T G C A T C C G C T A A T A 287

C. duobushaemulonii (JX459667.1) $5^{\prime}$ C C C A G G T G G T T T T G C A T C C G C T A A T A 2891

C.lusitaniae (NR_130677.1) 5 ' C C G G T T A G G C G T T G C T C C - - - G A A A T 2871

\section{B}

Clade I/III 5, A A A A C A A A A A C A TA A A A C T T T A A C A A C G G A T C T C T T G T $130^{1}$

Clade II $5{ }^{\prime}$ A A A C TA TA A A GA- - A A A C T T T CA A CA A C G A T C T C T T G T $128^{1}$ ITS_CauCIIR A T A T T T C T - T T T G A A A G T T G T T G C C A A A G

Clade IV 5' A A A C - - A A A A C A T A A A A C T T T C A A C A A C G G A T C T C T T G G T 1271 ITS_CauCIV/V_R G - - T T T G T A T T T T G A A A G T T G T T G C C T A G A

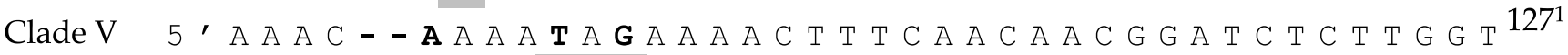
ITS_CauV_R

Figure 1. Allele-specific primer binding sites in the ITS region for the identification of C. auris (A) and C. auris clades (B). Specific polymorph nucleotides are shown in bold, primers are shown in gray, and mismatches contained within the primer are shown in red. ${ }^{1}$ Nucleotide position from ITS1 forward primer. ${ }^{2}$ Length based on ITS region of clade I.

\section{6. $A S-P C R$}

All AS-PCRs were performed using Taq DNA polymerase with Standard Taq buffer (New England Biolabs Inc.). The total reaction volume of $20 \mu \mathrm{L}$ contained $2 \mu \mathrm{L}$ of purified DNA extract $(10 \mathrm{ng} / \mu \mathrm{L}), 1.6 \mu \mathrm{L}$ of dNTPs $(2.5 \mathrm{mM}), 2 \mu \mathrm{L}$ of $10 \times$ Standard Taq buffer, $0.1 \mu \mathrm{L}$ of Taq DNA polymerase, and the following primers: $0.1 \mu \mathrm{L}$ of the ITS1 forward primer $(100 \mu \mathrm{M})$ and the ITS_Cau_R reverse primer $(100 \mu \mathrm{M})$ for species identification (simplex PCR) or $0.2 \mu \mathrm{L}$ of the ITS1 forward primer $(100 \mu \mathrm{M}), 0.1 \mu \mathrm{L}$ of the ITS_Cau_R reverse primer $(100 \mu \mathrm{M})$, and $0.2 \mu \mathrm{L}$ of the clade-specific reverse primer $(100 \mu \mathrm{M})$ for clade identification (duplex PCR). The PCR program consisted of initial denaturation at $95^{\circ} \mathrm{C}$ for $30 \mathrm{~min}, 35$ cycles of $95^{\circ} \mathrm{C}$ for $20 \mathrm{~min}$, primer annealing at a primer specific temperature (Ta, see Table 1) for $30 \mathrm{~min}$, and amplicon elongation for $30 \mathrm{~min}$ at $68^{\circ} \mathrm{C}$. The PCR was terminated by a final elongation at $68^{\circ} \mathrm{C}$ for $5 \mathrm{~min}$. All reactions were performed using a Labcycler Basic thermocycler (Bioké). Correct amplification was verified by $2 \%$ agarose gel electrophoresis of $10 \mu \mathrm{L}$ of the PCR product. The specific annealing temperature of all AS-primers was identified by performing the same procedure as described above but using a 12-step temperature gradient from $50^{\circ} \mathrm{C}$ to $70^{\circ} \mathrm{C}$ and $60^{\circ} \mathrm{C}$ to $80^{\circ} \mathrm{C}$ as annealing temperature. From the window of specific amplification, one temperature was selected as annealing temperature (Ta).

\section{Results}

\subsection{C. auris Identification}

The alignment of 32 species showed great variation within the ITS regions. Figure 1A shows a fragment of the ITS2 region on which the C. auris specific reverse primer (ITS_Cau_R) was designed, amplifying a fragment of 296-293 bp when paired with the universal fungal barcoding forward primer ITS1 [47]. The allele-specific reverse primer contains a G-T mismatch at the third position of the $3^{\prime}$ end to increase specificity for $C$. auris. At an annealing temperature of $78{ }^{\circ} \mathrm{C}, \mathrm{C}$. auris but not C. haemulonii, C. pseudohaemulonii, $\mathrm{C}$. duobushaemulonii, C. albicans, C. glabrata, C. dubliniensis, C. tropicalis, C. parapsilosis, C. orthopsilosis, C. metapsilosis, C. lustianae, C. krusei, C. sake, or S. cerevisiae DNA is amplified, as shown in Figure 2A. Sequencing the ITS region of our panel of 15 species confirmed 
the variability in the ITS2 region which was targeted. This region did not show variability between C. auris clades.
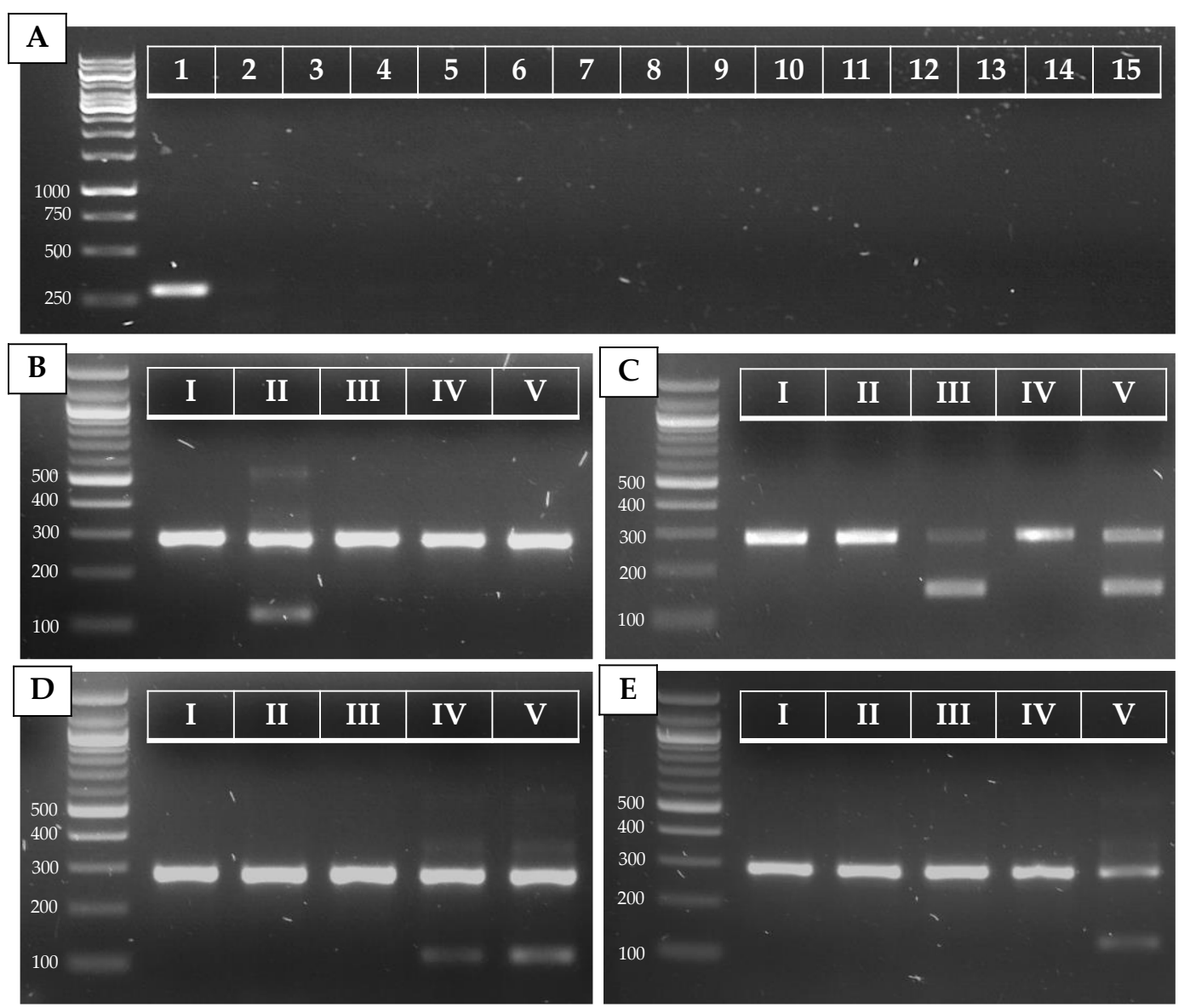

Figure 2. PCR results for C. auris (A) and C. auris clade (B-E) identification. (A) Simplex PCR of the ITS1-ITS_CauR primer pair at $78^{\circ} \mathrm{C}$ Ta on $20 \mathrm{ng}$ of purified DNA of C. auris (1), C. haemulonii (2), C. duobushaemulonii (3), C. pseudohaemulonii (4), C. albicans (5), C. tropicalis (6), C. parapsilosis (7), C. glabrata (8), C. lusitaniae (9), C. orthopsilosis (10), C. metapsilosis (11), C. sake (12), C. dubliniensis (13), C. krusei (14), and S. cerevisiae (15). (B-E) Duplex PCR of the ITS1-ITS_Cau_R and ITS1-ITS_CauCII_R (B), RHA1_CauCIII/V_F-RHA1_CauCIII/V_R (C), ITS1-ITS_CauCIV/V_R (D), and ITS1-ITS_CauCV_R (E) primer pairs at $68^{\circ} \mathrm{C}$ Ta on $20 \mathrm{ng}$ of purified DNA of C. auris B8441 (I), B11220 (II), B11221 (III), B11244 (IV), and B18474 (V), which are reference strains from clades I to $\mathrm{V}$, respectively.

\subsection{C. auris Clade Identification}

The ITS alignment of 121 C. auris strains from all five C. auris clades showed no intra-clade variability within the ITS region. Between clades, in both the ITS1 and the ITS2 regions, clade-specific polymorphisms were found. This resulted in four main ITS-based clusters (clades I and III, clade II, clade IV, and clade V), in a phylogenetic tree (Figure A1, Appendix A). A rhamnose assimilation gene cluster, reported to be present in clade III but deleted in clades I, II, and IV [46], was found to be present in the three clade V isolates that were investigated. Targeting this gene cluster and a clade V-specific ITS region enabled to discriminate clade III from clade I (Figure 2C,E).

Several strains from our in-house collection were typed on the basis of whole-genome sequencing data (e.g., strain 14 (B8441, clade I), strain 23 (B11220, clade II), strain 3 (B11221, clade III), strain 2 (B11245, clade IV), and strain 22 (B18474, clade V); see Table A2, Appendix A). We optimized and tested our AS-PCR primers using these strains and confirm correct clade identification (Figure 2B-E). Moreover, correct placement of the sequenced ITS region of these strains within the correct clade cluster reconfirmed clade phylogeny (Figure A1, Appendix A). 
To test our AS-PCR for clade identification, 23 C. auris strains (15 clade I strains, one clade II strain, three clade III strains, three clade IV strains, and one clade V strain) were screened. Information about these strains is summarized in Table A2 (Appendix A). The results of the four allele-specific multiplex PCRs in which the C. auris-specific amplicon (primers ITS1 and ITS_Cau_R) is duplexed with the clade II (primers ITS1 and ITS_CauCII_R)-, clade III and V (primers RHA1_CauCIII/V_F and RHA1_CauCIII/V_R)-, clade IV and V (primers ITS and ITS_CauCIV/V_R)-, and clade V (primers ITS1 and ITS_CauCV_R)-specific amplicons, is shown in Figure A2 (Appendix A). This shows that our multiplex AS-PCR assays for clade detection are $100 \%$ specific. Additionally, our multiplex design decreases the chance for false-negative results, as the C. auris-specific amplicon works as a positive control for successful PCR amplification.

\section{Discussion}

In this study, we show that combining the variability in the ITS region with the presence or absence of a rhamnose assimilation gene cluster can be used to identify C. auris and identify to which of the five main clades the $C$. auris strain belongs. The four ASPCR assays for clade identification each consist of a duplex PCR reaction with a C. aurisspecific amplicon and a clade-specific amplicon. This significantly reduces the chance of false-negative results in screening assays, as the $C$. auris-specific amplicon serves as an internal control. AS-PCR provides a rapid, low-cost, low-tech alternative to other clade identification methods such as (genome) sequencing or microsatellite typing. Nevertheless, we do recommend validating this diagnostic assay with sequencing and typed reference strains before use in screenings, as PCR-based methods can show variability due to technical discrepancies.

Molecular diagnostics are some of the most reliable identification methods for microorganisms. However, sequencing of molecular barcodes such as ITS requires time, specialized equipment, and analysis. Therefore, several sequencing-independent DNA-based diagnostic assays have been developed. These can be divided into three groups: end-point PCR assays (simplex or multiplex PCR and gel electrophoresis), quantitative PCR (qPCR) methods, and nonconventional detection methods such as loop-mediated isothermal amplification (LAMP) or T2 nuclear magnetic resonance measurement [9]. The method we report here belongs to the first group and only requires standard PCR reagents, a thermocycler, and gel electrophoresis setup. Such a method is ideally fit for low-budget research settings. Nevertheless, our method has some potential disadvantages. It is, like most diagnostic methods, a culture-dependent assay. Additionally, no non-auris Candida species can be detected, and typing is limited to the five currently described clades. Nevertheless, AS-PCR can be optimized as a qPCR assay for culture-independent diagnosis, as reported with other qPCR methods for detection in clinical samples [8], and other species-specific primers can be designed.

We used the ITS region for species identification as this is the primary fungal barcoding marker, proposed by the Fungal Barcoding Consortium [48]. The ITS region consists of two spacer sequences surrounding the 5.8S rRNA gene in the ribosomal cistron and allows successful identification of a broad range of fungi with a clearly defined barcoding gap between inter- and intraspecific variation [48]. Moreover, single-copy protein-coding regions often show lower PCR amplification and sequencing success compared to the multicopy ITS region, which yields a PCR amplification success of $100 \%$ for Saccharomycotina, the subphylum to which Candida species belong [48]. ITS sequencing analyses [4], as well as ITS-based diagnostic PCR assays [44,49,50], have been widely reported for C. auris identification. In addition to species-level identification, ITS sequencing has been used for typing $C$. auris and other fungal species in the past $[31,48,51]$. Here, we show that the ITS sequence harbors sufficient interclade diversity to discriminate four out of five clades. An L-rhamnose gene cluster was used to discriminate clade III from clade I, as they share the same ITS sequence. The L-rhamnose gene cluster contains seven genes (RHA1, LRA1, LRA2, LRA3, two copies of TRC1, and an MFS transporter) which are absent 
in clade I, II, and IV isolates but present in clade III [46]. This pattern was discovered by testing an updated Vitek 2 yeast identification system, in which all clade III isolates but hardly any other $C$. auris isolates or $C$. haemulonii showed the ability to assimilate L-rhamnose [46]. This phenotype has not been validated for clade $V$ isolates, but here we show that the L-rhamnose gene cluster is present in three clade V strains, including the type specimen [12].

Clade typing can be of significant epidemiological value, as it provides information on the origin of a strain and can help to monitor nosocomial transmission. Moreover, clade diagnosis can have a clinical value, as clade-associated virulence and resistance implications have been reported [2,3,12,17,20-27,30,52]. Identification of the clade to which C. auris isolates could belong in an outbreak might, thus, have implications for the choice of treatment and the use of infection control and prevention measures. In scientific research, clade identification is also essential. Molecular and pharmaceutical research of $C$. auris implies the use of isolates from different clades due to the outspoken phenotypic difference between clades and the possible implications for scientific conclusions on a species level $[2,3,17,20-27,30,52]$. Another useful purpose of screening isolates with this diagnostic AS-PCR assay is the potential to identify new clades. When the patterns of clade-specific PCRs differ from an expected outcome, this could be an indication of a novel, undescribed clade. Whole-genome sequencing and microsatellite typing will provide more in-depth insight in such circumstances. Detection of new clades could have a profound impact on our understanding of the emergence and epidemiology of this novel fungal pathogen.

In conclusion, we provide a molecular diagnostic assay to identify $C$. auris and the five currently described C. auris clades, which can be used for epidemiological, pharmaceutical, clinical, and molecular research. The low-cost and low-tech necessities and fast readout make it a convenient tool, ideal for low-budget research settings. As the number of reported C. auris cases and outbreaks is still on the rise, the use and development of up-to-date, reliable identification and typing tools is essential.

Author Contributions: Conceptualization, H.C.; methodology, H.C. and C.A.C.; validation, H.C., S.J. and C.L.R.; formal analysis, H.C., S.J. and Q.D.; investigation, H.C., S.J. and C.L.R.; resources, J.F.M. and P.V.D.; data curation, H.C.; writing-original draft preparation, H.C.; writing-review and editing, H.C., S.J., C.L.R., Q.D., C.A.C., J.F.M. and P.V.D.; supervision, H.C.; funding acquisition, P.V.D. All authors read and agreed to the published version of the manuscript.

Funding: This work was supported by a Fund for Scientific Research Flanders (FWO), personal research grant nr. 11D7620N awarded to H.C.

Institutional Review Board Statement: Not applicable.

Informed Consent Statement: Not applicable.

Data Availability Statement: All original data are available from the authors. No large datasets were generated.

Acknowledgments: We would like to thank Koos Albertyn (University of Free State, Bloemfontein, South Africa) and Katrien Lagrou (KU Leuven, Leuven, Belgium) for the donation of C. auris strains.

Conflicts of Interest: The authors declare no conflict of interest. 


\section{Appendix A}

Table A1. All species included in the alignment to identify a C. auris-specific ITS region for the design of primer ITS_Cau_R. The species and strain names are given, along with the ITS sequence NCBI GenBank accession and the size of the sequence in the alignment after trimming.

\begin{tabular}{|c|c|c|c|}
\hline Name & NCBI Accession Nr. & Strain & Size (bp) \\
\hline Candida auris & NR_154998.1 & CBS 10913 & 342 \\
\hline Candida haemulonii & JX45̄96664.1 & CBS 6590 & 479 \\
\hline Candida haemulonii var. vulnera & JX459687.1 & CNMCL7256 & 322 \\
\hline Candida pseudohaemulonii & EU881972.1 & C4368 & 327 \\
\hline Candida duobushaemulonii & JX459667.1 & CBS 7799 & 487 \\
\hline Candida albicans & NR_125332.1 & CBS 562 & 462 \\
\hline Candida tropicalis & NR_111250.1 & CBS 94 & 454 \\
\hline Candida lusitaniae & NR_130677.1 & CBS 6936 & 307 \\
\hline Candida glabrata & NR_130691.1 & NRRL Y-65 & 655 \\
\hline Candida dubliniensis & NR_119386.1 & CBS 7987 & 460 \\
\hline Candida parapsilosis & NR_130673.1 & ATCC 22019 & 461 \\
\hline Candida orthopsilosis & NR_130661.1 & ATCC 96139 & 436 \\
\hline Candida metapsilosis & NR_165186.1 & CBS 10907 & 472 \\
\hline Candida sake & NR_151807.1 & CBS 159 & 432 \\
\hline Candida inconspicua & NR_111116.1 & CBS 180 & 379 \\
\hline Candida castellii & NR_154958.1 & CBS 4332 & 628 \\
\hline Candida bracarensis & NR_136973.1 & CBS 10154 & 704 \\
\hline Candida pararugosa & MF797780.1 & S200 & 324 \\
\hline Candida guilliermondii (Meyerozyma guillermondii) & NR_111247.1 & CBS 2030 & 532 \\
\hline Candida krusei (Pichia kudriavzevii) & NR_131315.1 & ATCC 6258 & 435 \\
\hline Candida famata (Debaryomyces hansenii) & NR_120016.1 & JCM 1990 & 580 \\
\hline Candida kefyr (Kluyveromyces marxianus) & NR_111251.1 & CBS 712 & 628 \\
\hline Candida norvegensis (Pichia norvegensis) & MH396411.1 & URM 7762 & 434 \\
\hline Lodderomyces elongisporus & NR_111593.1 & ATCC 11503 & 496 \\
\hline Yarrowia lipolytica & NR_111212.1 & CBS 6124 & 285 \\
\hline Nakaseomyces delphensis & NR_167682.1 & CBS 2170 & 709 \\
\hline Nakaseomyces bacillisporus & NR_138202.1 & NRRL Y-17846 & 662 \\
\hline Kodameae ohmeri & NR_121464.1 & CBS 5367 & 348 \\
\hline Diutina catenulata & NR_077200.1 & CBS 565 & 343 \\
\hline Rhodotorula mucilaginosa (R. rubra) & NR_073296.1 & CBS 316 & 557 \\
\hline Rhodotorula glutinis & MH665424.1 & R.g0726 & 582 \\
\hline Saccharomyces cerevisiae & NR_111007.1 & CBS 1171 & 613 \\
\hline
\end{tabular}


Table A2. All C. auris strains used in this study to validate clade identification AS-PCRs. The strain name (if assigned), origin, clade, and reference to typing literature of the reference strains (bold) are indicated. Clade identification is based on ITS sequencing, microsatellite typing, and/or whole-genome sequencing.

\begin{tabular}{|c|c|c|c|c|}
\hline Nr. & Strain Name & Origin & Clade & $\begin{array}{l}\text { Typing } \\
\text { Reference }\end{array}$ \\
\hline 1 & B11244 & Venezuela & IV & {$[2,13,17]$} \\
\hline 2 & B11245 & Venezuela & IV & \\
\hline 3 & B11221 & South Africa & III & {$[2,3,13,17]$} \\
\hline 4 & B11222 & South Africa & III & \\
\hline 5 & CDC_390 & India & I & \\
\hline 6 & - & Colombia & IV & \\
\hline 7 & B11098 & Pakistan & $\mathrm{I}$ & \\
\hline 8 & B11203 & India & $\mathrm{I}$ & \\
\hline 9 & - & India & $\mathrm{I}$ & \\
\hline 10 & - & India & I & \\
\hline 11 & - & India & I & \\
\hline 12 & - & India & I & \\
\hline 13 & VPCI 714/P/14 & India & $\mathrm{I}$ & \\
\hline 14 & B8441 & Pakistan & I & {$[2,3,13,17]$} \\
\hline 15 & 317052804 & Oman & $\mathrm{I}$ & \\
\hline 16 & VPCI 514/P/14 & India & I & \\
\hline 17 & VPCI 1133/P/13 & India & I & \\
\hline 18 & B11109 & India & I & \\
\hline 19 & VPCI 1131/P/13 & India & I & \\
\hline 20 & OS299 & Belgium & I & \\
\hline 21 & MRU224 & South Africa & III & \\
\hline 22 & IFRC2087/B18474 & Iran & $\mathrm{V}$ & {$[12,17]$} \\
\hline 23 & B11220 & Japan & II & {$[2,3,13,17]$} \\
\hline
\end{tabular}




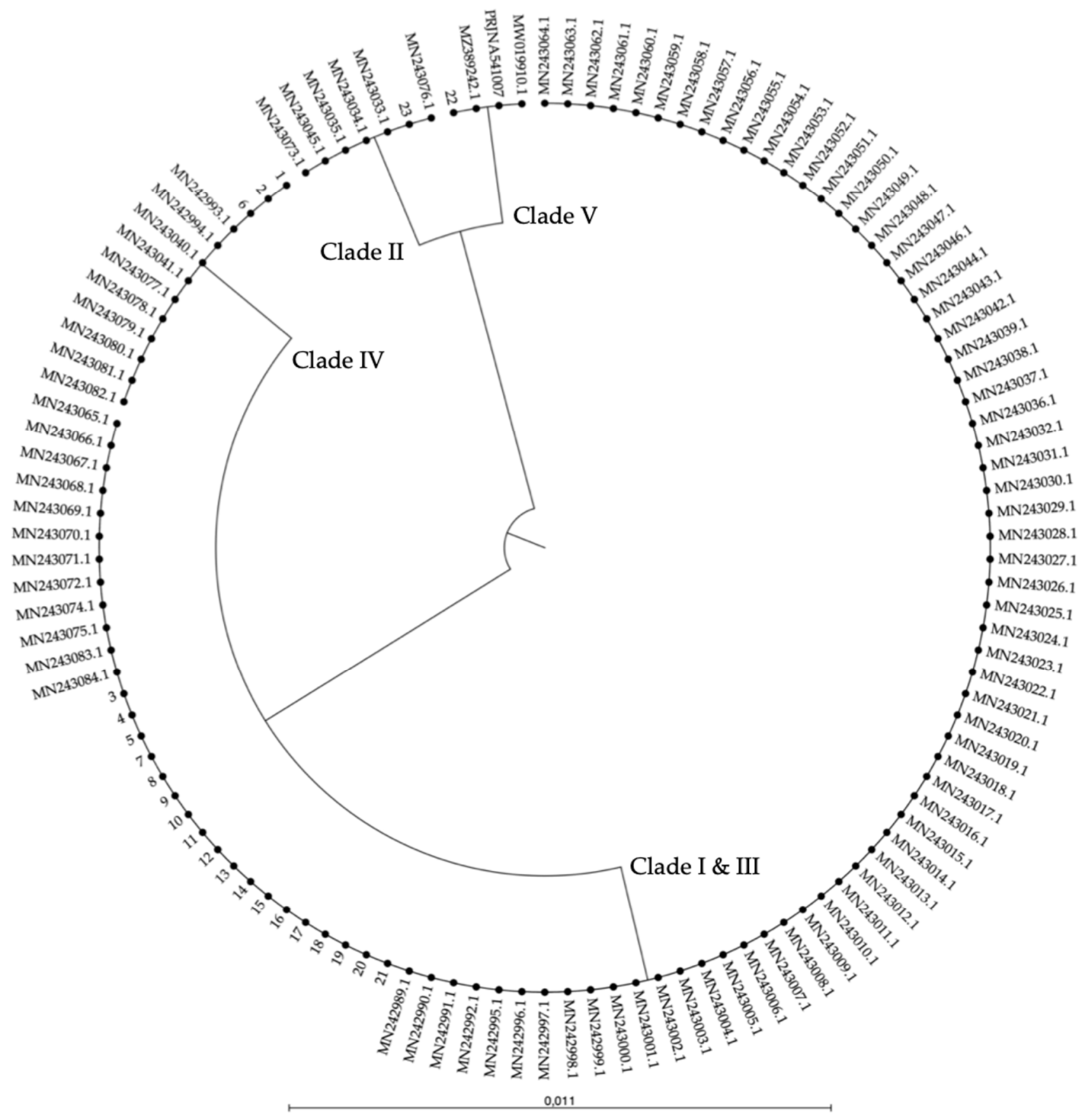

Figure A1. UPGMA dendrogram of a 248-251 bp ITS alignment of sequences of 121 C. auris strains: 96 typed strains from four clades [31] and three clade V strains [12,21,22], represented by their GenBank accessions, and 23 strains from our in-house collection, represented by the numbers 1 to 23 (see Table A2). Four ITS-based clusters are shown: clades I and III, clade II, clade IV, and clade V. The scale bar represents the percentage of nucleotide variation. 

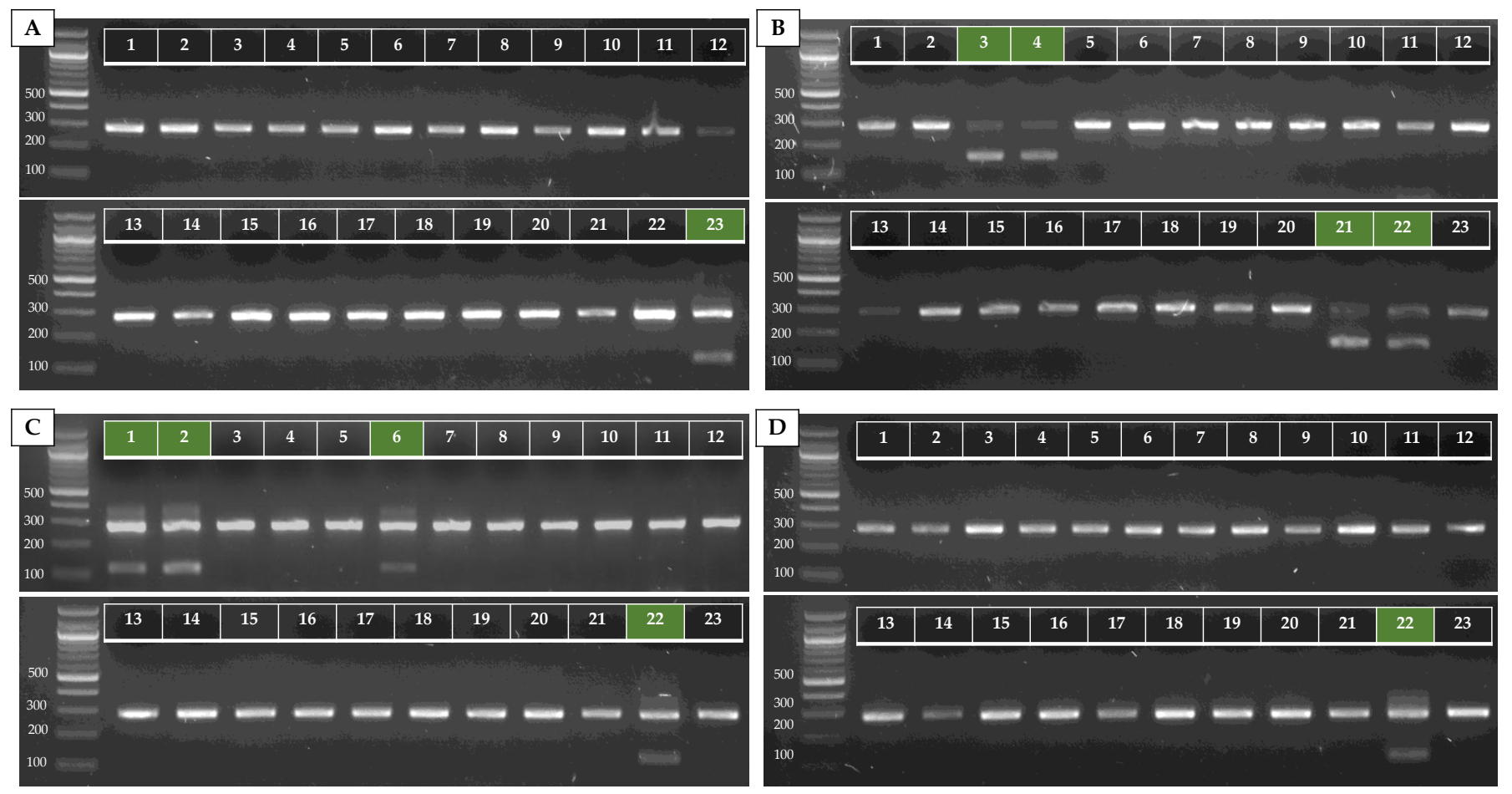

Figure A2. AS-PCR results of the four clade-specific PCRs for 23 strains of $C$. auris from our in-house collection, represented by the numbers 1-23 (see Table A2). Duplex PCRs of the ITS1-ITS_Cau_R and ITS1-ITS_CauCII_R (A), RHA1_CauCIII/V_FRHA1_CauCIII/V_R (B), ITS1-ITS_CauCIV/V_R (C), and ITS1-ITS_CauCV_R (D) primer pairs at $68^{\circ} \mathrm{C}$ Ta on $20 \mathrm{ng}$ of purified DNA are shown. The green markings indicate where a positive result should appear.

\section{References}

1. Satoh, K.; Makimura, K.; Hasumi, Y.; Nishiyama, Y.; Uchida, K.; Yamaguchi, H. Candida auris Sp. Nov., A Novel Ascomycetous Yeast Isolated from the External Ear Canal of an Inpatient in a Japanese Hospital. Microbiol. Immunol. 2009, 53, 41-44. [CrossRef] [PubMed]

2. Chow, N.A.; Muñoz, J.F.; Gade, L.; Berkow, E.; Li, X.; Welsh, R.M.; Forsberg, K.; Lockhart, S.R.; Adam, R.; Alanio, A.; et al. Tracing the Evolutionary History and Global Expansion of Candida auris Using Population Genomic Analyses. MBio 2020, 11, e03364-19. [CrossRef] [PubMed]

3. Muñoz, J.F.; Gade, L.; Chow, N.A.; Loparev, V.N.; Juieng, P.; Berkow, E.L.; Farrer, R.A.; Litvintseva, A.P.; Cuomo, C.A. Genomic Insights into Multidrug-Resistance, Mating and Virulence in Candida auris and Related Emerging Species. Nat. Commun. 2018, 9 , 5346. [CrossRef]

4. Kordalewska, M.; Perlin, D.S. Identification of Drug Resistant Candida auris. Front. Microbiol. 2019, 10, 1918. [CrossRef] [PubMed]

5. Dewaele, K.; Lagrou, K.; Frans, J.; Hayette, M.-P.; Vernelen, K. Hospital Laboratory Survey for Identification of Candida auris in Belgium. J. Fungi 2019, 5, 84. [CrossRef]

6. Buil, J.B.; van der Lee, H.A.; Curfs-Breuker, I.; Verweij, P.E.; Meis, J.F. External Quality Assessment Evaluating the Ability of Dutch Clinical Microbiological Laboratories to Identify Candida auris. J. Fungi 2019, 5, 94. [CrossRef] [PubMed]

7. CDC. Identification of Candida auris. Available online: https://www.cdc.gov/fungal/candida-auris/identification.html\#identify (accessed on 10 August 2021).

8. Caceres, D.H.; Forsberg, K.; Welsh, R.M.; Sexton, D.J.; Lockhart, S.R.; Jackson, B.R.; Chiller, T. Candida auris: A Review of Recommendations for Detection and Control in Healthcare Settings. J. Fungi 2019, 5, 111. [CrossRef]

9. Mahmoudi, S.; Afshari, S.A.K.; Gharehbolagh, S.A.; Mirhendi, H.; Makimura, K. Methods for Identification of Candida auris, the Yeast of Global Public Health Concern: A review. J. Mycol. Med. 2019, 29, 174-179. [CrossRef] [PubMed]

10. Sattler, J.; Noster, J.; Brunke, A.; Plum, G.; Wiegel, P.; Kurzai, O.; Meis, J.F.; Hamprecht, A. Comparison of Two Commercially Available qPCR Kits for the Detection of Candida auris. J. Fungi 2021, 7, 154. [CrossRef]

11. Martínez-Murcia, A.; Navarro, A.; Bru, G.; Chowdhary, A.; Hagen, F.; Meis, J.F. Internal Validation of GPS ${ }^{\mathrm{TM}}$ MONODOSE CanAur Dtec-qPCR Kit Following the UNE/EN ISO/IEC 17025: 2005 for Detection of the Emerging Yeast Candida auris. Mycoses 2018, 61, 877-884. [CrossRef]

12. Chow, N.A.; de Groot, T.; Badali, H.; Abastabar, M.; Chiller, T.M.; Meis, J.F. Potential Fifth Clade of Candida auris, Iran, 2018. Emerg. Infect. Dis. 2019, 25, 1780-1781. [CrossRef] 
13. Lockhart, S.R.; Etienne, K.A.; Vallabhaneni, S.; Farooqi, J.; Chowdhary, A.; Govender, N.P.; Colombo, A.L.; Calvo, B.; Cuomo, C.A.; Desjardins, C.A.; et al. Simultaneous Emergence of Multidrug-Resistant Candida auris on 3 Continents Confirmed by Whole-Genome Sequencing and Epidemiological Analyses. Clin. Infect. Dis. 2017, 64, 134-140. [CrossRef] [PubMed]

14. CDC. Tracking Candida auris. Available online: https://www.cdc.gov/fungal/candida-auris/tracking-c-auris.html (accessed on 10 August 2021).

15. Kean, R.; Brown, J.; Gulmez, D.; Ware, A.; Ramage, G. Candida auris: A Decade of Understanding of an Enigmatic Pathogenic Yeast. J. Fungi 2020, 6, 30. [CrossRef] [PubMed]

16. Bruno, M.; Kersten, S.; Bain, J.M.; Jaeger, M.; Rosati, D.; Kruppa, M.D.; Lowman, D.W.; Rice, P.J.; Graves, B.; Ma, Z.; et al. Transcriptional and Functional Insights into the Host Immune Response against the Emerging Fungal Pathogen Candida auris. Nat. Microbiol. 2020, 5, 1516-1531. [CrossRef]

17. Muñoz, J.F.; Welsh, R.M.; Shea, T.; Batra, D.; Gade, L.; Howard, D.; Rowe, L.A.; Meis, J.F.; Litvintseva, A.P.; Cuomo, C.A. Clade-Specific Chromosomal Rearrangements and Loss of Subtelomeric Adhesins in Candida auris. Genetics 2021, 218 , iyab029. [CrossRef] [PubMed]

18. Chowdhary, A.; Prakash, A.; Sharma, C.; Kordalewska, M.; Kumar, A.; Sarma, S.; Tarai, B.; Singh, A.; Upadhyaya, G.; Upadhyay, S.; et al. A Multicentre Study of Antifungal Susceptibility Patterns among 350 Candida auris Isolates (2009-2017) in India: Role of the ERG11 and FKS1 Genes in Azole and Echinocandin Resistance. J. Antimicrob. Chemother. 2018, 73, 891-899. [CrossRef]

19. Maphanga, T.G.; Naicker, S.D.; Kwenda, S.; Muñoz, J.F.; van Schalkwyk, E.; Wadula, J.; Nana, T.; Ismail, A.; Coetzee, J.; Govind, C. In-Vitro Antifungal Resistance of Candida auris Isolates from Bloodstream Infections, South Africa. Antimicrob. Agents Chemother. 2021, 65, e00517-21. [CrossRef] [PubMed]

20. Welsh, R.M.; Sexton, D.J.; Forsberg, K.; Vallabhaneni, S.; Litvintseva, A. Insights into the Unique Nature of the East Asian Clade of the Emerging Pathogenic Yeast Candida auris. J. Clin. Microbiol. 2019, 57, e00007-19. [CrossRef] [PubMed]

21. Safari, F.; Madani, M.; Badali, H.; Kargoshaie, A.; Fakhim, H.; Kheirollahi, M.; Meis, J.F.; Mirhendi, H. Autochthonous Fifth Clade Case of Candida auris Otomycosis in Iran. Mycopathologia 2021, in press.

22. Taghizadeh Armaki, M.; Mahdavi Omran, S.; Kiakojuri, K.; Khojasteh, S.; Jafarzadeh, J.; Tavakoli, M.; Badali, H.; Haghani, I.; Shokohi, T.; Hedayati, M.T. First Fluconazole-Resistant Candida auris Isolated from Fungal Otitis in Iran. Curr. Med. Mycol. 2021, 7, 51-54.

23. Sexton, D.J.; Welsh, R.M.; Bentz, M.L.; Forsberg, K.; Jackson, B.; Berkow, E.L.; Litvintseva, A.P. Evaluation of Nine Surface Disinfectants against Candida auris Using a Quantitative Disk Carrier Method: EPA SOP-MB-35. Infect. Control Hosp. Epidemiol. 2020, 41, 1219-1221. [CrossRef]

24. Chatterjee, P.; Choi, H.; Ochoa, B.; Garmon, G.; Coppin, J.D.; Allton, Y.; Lukey, J.; Williams, M.D.; Navarathna, D.; Jinadatha, C. Clade-Specific Variation in Susceptibility of Candida auris to Broad-Spectrum Ultraviolet C light (UV-C). Infect. Control Hosp. Epidemiol. 2020, 41, 1384-1387. [CrossRef] [PubMed]

25. De Groot, T.; Chowdhary, A.; Meis, J.F.; Voss, A. Killing of Candida auris by UV-C: Importance of Exposure Time and Distance. Mycoses 2019, 62, 408-412. [CrossRef]

26. Abe, M.; Katano, H.; Nagi, M.; Higashi, Y.; Sato, Y.; Kikuchi, K.; Hasegawa, H.; Miyazaki, Y. Potency of Gastrointestinal Colonization and Virulence of Candida auris in a Murine Endogenous Candidiasis. PLoS ONE 2020, 15, e0243223. [CrossRef] [PubMed]

27. Forgács, L.; Borman, A.M.; Prépost, E.; Tóth, Z.; Kardos, G.; Kovács, R.; Szekely, A.; Nagy, F.; Kovacs, I.; Majoros, L. Comparison of In Vivo Pathogenicity of Four Candida auris Clades in a Neutropenic Bloodstream Infection Murine Model. Emerg. Microbes Infect. 2020, 9, 1160-1169. [CrossRef] [PubMed]

28. Short, B.; Brown, J.; Delaney, C.; Sherry, L.; Williams, C.; Ramage, G.; Kean, R. Candida auris Exhibits Resilient Biofilm Characteristics In Vitro: Implications for Environmental Persistence. J. Hosp. Infect. 2019, 103, 92-96. [CrossRef]

29. Chatzimoschou, A.; Giampani, A.; Meis, J.F.; Roilides, E. Activities of Nine Antifungal Agents Against Candida auris Biofilms. Mycoses 2021, 64, 381-384. [CrossRef] [PubMed]

30. Brown, J.L.; Delaney, C.; Short, B.; Butcher, M.C.; McKloud, E.; Williams, C.; Kean, R.; Ramage, G. Candida auris Phenotypic Heterogeneity Determines Pathogenicity In Vitro. Msphere 2020, 5, e00371-20. [CrossRef]

31. Vatanshenassan, M.; Boekhout, T.; Mauder, N.; Robert, V.; Maier, T.; Meis, J.F.; Berman, J.; Then, E.; Kostrzewa, M.; Hagen, F. Evaluation of Microsatellite Typing, ITS Sequencing, AFLP Fingerprinting, MALDI-TOF MS, and Fourier-Transform Infrared Spectroscopy Analysis of Candida auris. J. Fungi 2020, 6, 146. [CrossRef] [PubMed]

32. De Groot, T.; Puts, Y.; Berrio, I.; Chowdhary, A.; Meis, J.F. Development of Candida auris Short Tandem Repeat Typing and Its Application to a Global Collection of Isolates. MBio 2020, 11, e02971-19. [CrossRef]

33. Rhodes, J.; Fisher, M.C. Global Epidemiology of Emerging Candida auris. Curr. Opin. Microbiol. 2019, 52, 84-89. [CrossRef] [PubMed]

34. Liu, J.; Huang, S.; Sun, M.; Liu, S.; Liu, Y.; Wang, W.; Zhang, X.; Wang, H.; Hua, W. An Improved Allele-Specific PCR Primer Design Method for SNP Marker Analysis and Its Application. Plant Methods 2012, 8, 34. [CrossRef]

35. Carolus, H.; Pierson, S.; Muñoz, J.F.; Subotić, A.; Cruz, R.B.; Cuomo, C.A.; Van Dijck, P. Genome-Wide Analysis of Experimentally Evolved Candida auris Reveals Multiple Novel Mechanisms of Multidrug Resistance. Mbio 2021, 12, e03333-20. [CrossRef] 
36. Hou, X.; Lee, A.; Jiménez-Ortigosa, C.; Kordalewska, M.; Perlin, D.S.; Zhao, Y. Rapid Detection of ERG11-Associated Azole Resistance and FKS-Associated Echinocandin Resistance in Candida auris. Antimicrob. Agents Chemother. 2019, 63, e01811-18. [CrossRef] [PubMed]

37. Ding, X.; Wang, Z.; Zhou, K.; Xu, L.; Xu, H.; Wang, Y. Allele-Specific Primers for Diagnostic PCR Authentication of Dendrobium officinale. Planta Med. 2003, 69, 587-588. [PubMed]

38. Wu, D.Y.; Ugozzoli, L.; Pal, B.K.; Wallace, R.B. Allele-Specific Enzymatic Amplification of Beta-Globin Genomic DNA for Diagnosis of Sickle Cell Anemia. Proc. Natl. Acad. Sci. USA 1989, 86, 2757-2760. [CrossRef]

39. Weber, B.; Meldgaard, P.; Hager, H.; Wu, L.; Wei, W.; Tsai, J.; Khalil, A.; Nexo, E.; Sorensen, B.S. Detection of EGFR Mutations in Plasma and Biopsies from Non-Small Cell Lung Cancer Patients by Allele-Specific PCR Assays. BMC Cancer 2014, 14, 294. [CrossRef]

40. Sanchaisuriya, K.; Chunpanich, S.; Fucharoen, G.; Fucharoen, S. Multiplex Allele-Specific PCR Assay for Differential Diagnosis of $\mathrm{Hb}$ S, Hb D-Punjab and $\mathrm{Hb}$ Tak. Clin. Chim. Acta 2004, 343, 129-134. [CrossRef]

41. Lone, S.A.; Ahmad, A. Candida auris-The Growing Menace to Global Health. Mycoses 2019, 62, 620-637. [CrossRef] [PubMed]

42. Mizusawa, M.; Miller, H.; Green, R.; Lee, R.; Durante, M.; Perkins, R.; Hewitt, C.; Simner, P.J.; Carroll, K.C.; Hayden, R.T.; et al. Can Multidrug-Resistant Candida auris Be Reliably Identified in Clinical Microbiology Laboratories? J. Clin. Microbiol. 2017, 55, 638-640. [CrossRef]

43. Snayd, M.; Dias, F.; Ryan, R.W.; Clout, D.; Banach, D.B.; Warnock, D.W. Misidentification of Candida auris by RapID Yeast Plus, a Commercial, Biochemical Enzyme-Based Manual Rapid Identification System. J. Clin. Microbiol. 2018, 56, e00080-18. [CrossRef] [PubMed]

44. Theill, L.; Dudiuk, C.; Morales-Lopez, S.; Berrio, I.; Rodríguez, J.Y.; Marin, A.; Gamarra, S.; Garcia-Effron, G. Single-tube classical PCR for Candida auris and Candida haemulonii Identification. Rev. Iberoam Micol 2018, 35, 110-112. [CrossRef]

45. Turner, S.A.; Butler, G. The Candida Pathogenic Species Complex. Cold Spring Harb. Perspect. Med. 2014, 4, a019778. [CrossRef]

46. Ambaraghassi, G.; Dufresne, P.J.; Dufresne, S.F.; Vallières, E.; Muñoz, J.F.; Cuomo, C.A.; Berkow, E.L.; Lockhart, S.R.; Luong, M.-L. Identification of Candida auris by Use of the Updated Vitek 2 Yeast Identification System, Version 8.01: A Multilaboratory Evaluation Study. J. Clin. Microbiol. 2019, 57, e00884-19. [CrossRef] [PubMed]

47. White, T.J.; Bruns, T.; Lee, S.; Taylor, J. Amplification and Direct Sequencing of Fungal Ribosomal RNA Genes for Phylogenetics. PCR Protoc. Guide Methods Appl. 1990, 18, 315-322.

48. Schoch, C.L.; Seifert, K.A.; Huhndorf, S.; Robert, V.; Spouge, J.L.; Levesque, C.A.; Chen, W.; Consortium, F.B. Nuclear Ribosomal Internal Transcribed Spacer (ITS) Region as a Universal DNA Barcode Marker for Fungi. Proc. Natl. Acad. Sci. USA 2012, 109, 6241-6246. [CrossRef] [PubMed]

49. Leach, L.; Zhu, Y.; Chaturvedi, S. Development and Validation of a Real-Time PCR Assay for Rapid Detection of Candida auris from Surveillance Samples. J. Clin. Microbiol. 2018, 56, e01223-17. [CrossRef]

50. Walchak, R.C.; Buckwalter, S.P.; Zinsmaster, N.M.; Henn, K.M.; Johnson, K.M.; Koelsch, J.M.; Herring, S.A.; Steinmetz, L.K.; Reed, K.A.; Barth, J.E. Candida auris Direct Detection from Surveillance Swabs, Blood, and Urine Using a Laboratory-Developed PCR Method. J. Fungi 2020, 6, 224. [CrossRef]

51. Prakash, A.; Sharma, C.; Singh, A.; Singh, P.K.; Kumar, A.; Hagen, F.; Govender, N.; Colombo, A.; Meis, J.; Chowdhary, A. Evidence of Genotypic Diversity Among Candida auris Isolates by Multilocus Sequence Typing, Matrix-Assisted Laser Desorption Ionization Time-of-Flight Mass Spectrometry and Amplified Fragment Length Polymorphism. Clin. Microbiol. Infect. 2016, 22, 271-277. [CrossRef]

52. Szekely, A.; Borman, A.M.; Johnson, E.M. Candida auris Isolates of the Southern Asian and South African Lineages Exhibit Different Phenotypic and Antifungal Susceptibility Profiles In Vitro. J. Clin. Microbiol. 2019, 57, e02055-18. [CrossRef] [PubMed] 